\title{
Gene-chip studies of adipogenesis-regulated microRNAs in mouse primary adipocytes and human obesity
}

Pernille Keller ${ }^{4}$, Valentina Gburcik ${ }^{1}$, Natasa Petrovic ${ }^{2}$, lain J Gallagher ${ }^{1,3}$, Jan Nedergaard ${ }^{2}$, Barbara Cannon ${ }^{1,2}$ and James A Timmons ${ }^{1,2^{*}}$

\begin{abstract}
Background: Adipose tissue abundance relies partly on the factors that regulate adipogenesis, i.e. proliferation and differentiation of adipocytes. While components of the transcriptional program that initiates adipogenesis is wellknown, the importance of microRNAs in adipogenesis is less well studied. We thus set out to investigate whether miRNAs would be actively modulated during adipogenesis and obesity.

Methods: Several models exist to study adipogenesis in vitro, of which the cell line 3T3-L1 is the most well known, albeit not the most physiologically appropriate. Thus, as an alternative, we produced EXIQON microarray of brown and white primary murine adipocytes (prior to and following differentiation) to yield global profiles of miRNAs.

Results: We found 65 miRNAs regulated during in vitro adipogenesis in primary adipocytes. We evaluated the similarity of our responses to those found in non-primary cell models, through literature data-mining. When comparing primary adipocyte profiles, with those of cell lines reported in the literature, we found a high degree of difference in 'adipogenesis' regulated miRNAs suggesting that the model systems may not be accurately representing adipogenesis. The expression of 10 adipogenesis-regulated miRNAs were studied using real-time qPCR and then we selected 5 miRNAs, that showed robust expression, were profiled in subcutaneous adipose tissue obtained from 20 humans with a range of body mass indices (BMI, range $=21-48$, and all samples have U133+2 Affymetrix profiles provided). Of the miRNAs tested, mir-21 was robustly expressed in human adipose tissue and positively correlated with BMI $(R 2=0.49, p<0.001)$.

Conclusion: In conclusion, we provide a preliminary analysis of miRNAs associated with primary cell in vitro adipogenesis and demonstrate that the inflammation-associated miRNA, mir-21 is up-regulated in subcutaneous adipose tissue in human obesity. Further, we provide a novel transcriptomics database of EXIQON and Affymetrix adipocyte profiles to facilitate data mining.
\end{abstract}

Keywords: primary white and brown adipocytes microRNAs, microarray, EXIQON, Affymetrix, Adipose tissue: adipocyte, transcriptome

\section{Background}

Obesity is a major global health problem linked to serious medical conditions including diabetes, heart disease, arthritis and cancer [1]. Adipose tissue is not only a main site of energy storage but also an important endocrine organ. It is a crucial regulator of energy balance and glucose homeostasis in mammals (reviewed in [2]).

\footnotetext{
* Correspondence: jamie.timmons@gmail.com

${ }^{1}$ Royal Veterinary College, University of London, UK

Full list of author information is available at the end of the article
}

Imbalances in energy homeostasis cause obesity. Most of the lipid reserves in the human body are stored in white adipose tissue (WAT) that predominates in adult humans. Brown adipose tissue (BAT), despite its ability to accumulate lipids, has a role not in storing but in converting fat into heat. Uncoupling Protein 1 (UCP-1) in the inner membrane of brown-fat mitochondria uncouples electron transport from ATP production, allowing energy dissipation, thus helping to regulate body temperature [3]. Recent evidence indicates that brown fat is
() Biomed Central

(c) 2011 Keller et al; licensee BioMed Central Ltd. This is an Open Access article distributed under the terms of the Creative Commons Attribution License (http://creativecommons.org/licenses/by/2.0), which permits unrestricted use, distribution, and reproduction in any medium, provided the original work is properly cited. 
important not only in newborns as previously thought but also in adult humans [4]. It has been suggested recently that human obesity is associated with altered function of brown adipose tissue [5,6] or appearance of brown adipocytes within WAT [7]. Understanding the regulation of the pathways that lead to proliferation and differentiation of white and brown pre-adipocytes could be crucial for revealing the underlying mechanisms of obesity. While primary white and brown pre-adipocytes look identical, these two cell types originate from separate precursor cells $[8]$ in the early embryo $[9,10]$. Brown fat cells arise from a pre-muscle cell lineage [8]. In addition to the classic brown adipocytes, a different type of brown fat cells seems to exist in tissues where WAT predominates. These cells are more closely related to white adipocytes but have the potential to induce UCP1 expression [11]. It has been suggested that adipogenesis is regulated by PPAR $\beta / \delta$ followed by PPAR $\gamma$ and $\mathrm{C} / \mathrm{EBP} \alpha$ promoting differentiation into mature adipocytes [12]. Maturation of BAT and WAT follow a similar adipogenic transcriptional program, albeit several genes show cell type-dependent expression. For instance, Prdm16 is expressed in BAT and when ectopically expressed in white preadipocytes, can promote brown fat differentiation [13].

A growing body of evidence implicates a role for microRNAs (miRNAs) in adipogenesis and obesity $[14,15]$. Indeed, the lineage difference between white and brown adipocytes has not only been shown on the basis of different mRNA expression but also at the levels of microRNA expression, as shown by Walden et al. [16]. Further, miRNAs can both target [17] and be induced by transcription factors [18]. MiRNAs are short non-coding RNA molecules that allow for fine-tuning of protein expression in vivo [19]. They act at a post-transcriptional level by binding to the 3' untranslated region (UTR) of an mRNA largely leading to translational regulation in vivo in mammals $[19,20]$. There are several examples of miRNAs that regulate metabolic processes associated with metabolic disease. Mir-122 can regulate lipid/glucose homeostasis in the liver [21]. Mir-375 [22] and miR-9 [23] can influence insulin secretion, while mir-34a can increase hormone secretion in insulin-secreting cells when exposed to palmitate [24]. The first miRNA found to play a role in maturation of human adipocytes was miR-143 [25,26]. miR-143 and also miR-103 can induce adipogenesis in 3T3-L1 adipocytes and augment or accelerate expression of several key adipogenesis-regulated genes, such as FABP4 and adiponectin [27]. Activation of the miRNA cluster miR-17 92 enhances adipogenesis [28] while let-7 and miR-27 impair adipogenic differentiation $[29,30]$. Several miRNAs are upregulated in adipose tissue in animal models of metabolic disease and/or obesity, e.g. mir-125a [31], mir29 [32], and mir-143 [33], while adipose tissue miR-519d expression is associated with human obesity [15]. Recently, Ortega et al. claimed that the expression of 70 miRNAs is modulated during differentiation of human adipocytes, with 15 of them showing dysregulation in obese individuals compared with non-obese persons [14].

Expression profiling of miRNAs in various adipocyte culture systems has yielded inconsistent results so far [25-27]. Studies have mostly been performed using cell lines and thus there is a lack of knowledge of the miRNA expression profile in primary adipocyte cells. Furthermore, there is no parallel analysis of both white and brown differentiating primary adipocytes. Thus, we performed a global expression profiling of miRNAs in white and brown murine primary cell cultures before and after differentiation. We found that 51 unique miRNAs were significantly upregulated and 14 significantly downregulated after the differentiation into adipocytes. We also demonstrated that differences in miRNA regulation between white and brown adipocytes during maturation are surprisingly modest. We investigated the expression of selected maturation-associated miRNAs in subcutaneous adipose tissue from humans with varying body mass indices and found that mir-21 expression correlated positively with human obesity. The present manuscript presents these miRNA gene-chip data sets, along with a pilot Affymetrix data set of $n=33$ human adipose global U133+2 tissue profiles, as a novel obesity and adipogenesis database for others to utilise.

\section{Methods}

\section{Murine samples}

The experiments were approved by the ethical committee of North Stockholm. Male outbred NMRI mice, 4 weeks old, were used for the preparation of primary cultures of brown and white preadipocytes, as described previously $[11,34]$. Mouse white adipose tissue (WAT) was harvested from the epididymal fat pad, while mouse brown adipose tissue (BAT) was harvested from the interscapular region. Preadipocytes were differentiated for 3 days (preadipocyte population) and 7 days (mature adipocyte population) in vitro. The culture medium was Dulbecco's modified Eagle's medium with $10 \%(\mathrm{v} / \mathrm{v})$ newborn calf serum (Invitrogen), $2.4 \mathrm{~nm}$ insulin, $25 \mu \mathrm{g} / \mathrm{ml}$ sodium ascorbate, $10 \mathrm{~mm}$ HEPES, $4 \mathrm{~mm}$ glutamine, 50 units $/ \mathrm{ml}$ penicillin, and $50 \mu \mathrm{g} / \mathrm{ml}$ streptomycin. The cells were grown at $37^{\circ} \mathrm{C}$ in an atmosphere of $8 \% \mathrm{CO}_{2}$ in air with $80 \%$ humidity. The medium was changed on day 1 and then every second day. RNA was extracted from preadipocytes and adipocytes using Trizol (Invitrogen), according to the manufacturer's instructions.

\section{Human samples and physiological measurements}

Twenty healthy subjects of Scandinavian origin with normal glucose tolerance but different degrees of obesity 
participated in the study conducted by CIM, Copenhagen. The study included individuals from a previous analysis [35] and individual data can be found along with global transcript profiles at GSE27951. Their mean (SD) age, BMI and $\mathrm{VO}_{2 \max }$ were 46 (13) years, 32 (7) $\mathrm{kg} / \mathrm{m}^{2}$ and $31(13) \mathrm{VO}_{2 \max } / \mathrm{kg}$, with the range in BMI being 21-48 $\mathrm{kg} / \mathrm{m}^{2}$. Adipose tissue biopsies were obtained from the subcutaneous abdominal region (from the area below the umbilicus in a $4-\mathrm{cm}$ range on either side) using the percutaneous needle biopsy technique with suction. Biopsies were quickly dissected free from visible blood and connective tissue and frozen in liquid nitrogen. To avoid interference of circadian rhythms and post-prandial responses, biopsies were obtained between 8 am and 10 am following an overnight fast. A general health examination and an oral glucose tolerance test (OGTT) were performed as previously described [35]. All tissue processing and RNA work was done independently of CIM, Copenhagen and CIM had no input on data analysis.

\section{RNA extraction and quality}

Frozen tissue was homogenised in $\operatorname{Trizol}^{\mathrm{TM}}$ (Invitrogen) using a motor-driven homogenizer (Polytron, Kinematica, Newark, NY, USA). RNA was extracted according to manufacturer's protocol, as previously described [35]. The quality of the total RNA was examined on an Agilent Bioanalyzer. Human adipose tissue RNA for miRNA Taqman qPCR assays was of good quality with spectrophotometer readings of A260/280 >1.7 and RIN scores $>7.5$. Murine RNA had A260/280 >1.9 and RIN scores $>8$.

\section{Exiqon miRCURYTM miRNA arrays}

Global microRNA expression analysis was performed using Exiqon (Vedbæk, Denmark) pre-spotted miRCURYTM V8.1 LNA microRNA microarrays. Preadipocyte and mature adipocyte samples $(n=3+3$, for brown adipocytes and $n=3+3$ for white adipocytes) were run individually on the arrays, i.e. cell cultures were not pooled. The arrays consist of 1458 capture probes (or as annotated on the GEO files) that are enhanced using locked nucleic acid (LNA) technology to normalize the Tm of the capture probes, as insertion of 1 LNA molecule into the capture probes increases the Tm by $2-8^{\circ} \mathrm{C}$. Total RNA was labelled with $\mathrm{Hy}^{3}$-dye using Exiqon's miRCURY LNA array labelling kit (Vedbæk, Denmark) with the inclusion of the miRCURY LNA array synthetic spike-in miRNAs in the labelling reaction. Two $\mu \mathrm{g}$ of total RNA was incubated with the $\mathrm{Hy}^{3}$ dye, labelling enzyme and spike-in microRNAs for 1 hour at $0^{\circ} \mathrm{C}$. The enzyme was then heat-inactivated at $65^{\circ} \mathrm{C}$ for $15 \mathrm{~min}$ utes, followed by addition of $2 \mathrm{x}$ hybridisation buffer. The samples were incubated at $95^{\circ} \mathrm{C}$ for 5 minutes, protected from light. The samples were then briefly spun down and filtered through a 0.45 -micron durapore filter (Millipore), and $40 \mu \mathrm{l}$ sample was loaded to the arrays by capillary force using a cover slip (Erie Scientific). The arrays were incubated at $60^{\circ} \mathrm{C}$ for 16 hours, then washed briefly in $60^{\circ} \mathrm{C}$ wash buffer $\mathrm{A}$, rinsed in wash buffer $\mathrm{B}$ followed by a 2-minute wash in wash buffer $B$ and a 2-minute wash in wash buffer $C$, according to the manufacturer's instructions. For drying the arrays, they were spun for 2 minutes at $1000 \mathrm{rpm}$ followed by immediate scanning using an Agilent G2565BA microarray scanner. Data were analyzed by QuantArray ${ }^{\circledR}$ software using the fixed-circle approach. Background subtracted signal intensities were normalized in $\mathrm{R}$ using quantile normalization. Data were then analysed using SAM analysis as previously described [19]. In this case on relevant murine probes and signals above background were utilised in the SAM analysis. The analysis to determine adipogenesis related miRNAs was performed using brown-cell and white-cell gene chips combined, to increase the sample size and hence statistical power. A selected subset of miRNAs modulated more than 1.5-fold was selected for further analysis by real-time qPCR.

\section{qPCR for the detection of microRNA expression levels in adipocytes}

The expression of 10 miRNAs that showed differential expression on the microarrays were measured in 12 RNA samples (3 preadipocyte and 3 adipocyte samples from both brown and white fat) using the Taqman ${ }^{\circledR}$ MicroRNA assays (Applied Bioystems) that detect mature miRNA. $10 \mathrm{ng}$ of total RNA was reverse transcribed using the microRNA-specific looped primer and the TaqMan ${ }^{\circledR}$ MicroRNA Reverse Transcription Kit (Applied Biosystems, PN 4366597), according to manufacturer's instructions. Thermal cycling conditions were as follows: $16^{\circ} \mathrm{C}$ for $30 \mathrm{~min}, 42^{\circ} \mathrm{C}$ for $30 \mathrm{~min}$, and $85^{\circ} \mathrm{C}$ for $5 \mathrm{~min}$. For qPCR, the TaqMan ${ }^{\circledR}$ 2X Universal PCR Master Mix, No AmpErase ${ }^{\circledR}$ UNG was used (Applied Biosystems, PN 4324020). Experiments were performed in triplicates on a 7900HT Fast Real-Time PCR System (Applied Biosystems), 9600 emulation mode. The Real-Time cycling conditions were: $95^{\circ} \mathrm{C}$ for 10 minutes followed by 50 cycles of $95^{\circ} \mathrm{C}$ for $15 \mathrm{sec}$ and $60^{\circ} \mathrm{C}$ for $1 \mathrm{~min}$. The expression of the reference RNAs used for normalisation, snoRNA142 (murine samples) and RNU48 (human samples), did not show variation between samples or subjects. All reactions were run single-plex and analysed and quantified using the $\Delta \Delta \mathrm{Ct}$ method. Data are expressed as a fold change from the value in non-obese persons for the human samples or as a fold change from preadipocyte-levels for the murine samples. miRNA data obtained by qPCR were analysed using a t-test to compare differences between $\Delta \mathrm{Ct}$ values ( $\mathrm{Ct}$ value of target miRNA minus $\mathrm{Ct}$ value of reference RNA) either between obese and non-obese persons, 
or between adipocytes and preadipocytes. For all analyses, $P<0.05$ was considered significant.

\section{qPCR for the detection of microRNA expression levels in human fat}

Of the 10 miRNAs that showed expression in the adipocyte cultures, we chose a subset of 5 miRNAs (mir-34c, mir-143, mir-24, mir-720 and mir-21) to measure in human adipose tissue RNA samples from obese persons $(\mathrm{BMI}>30, \mathrm{n}=10)$ and non-obese persons (BMI $<30$, $\mathrm{n}=10)$. The phenotype of these subjects has been described elsewhere $[7,19]$ and the subject demographics can be found, along with global mRNA transcriptome data at GSE27951. This data represents a large pilot study of $n=33$ human adipose tissue samples. We have previously reported selected gene changes in this tissue cohort. The RNA profile of the tissue did not support the conclusion that metabolic or mitochondrial genes were down-regulated in human obese adipose tissue and thus contrasts with Dahlman et al [36]. Dahlman et al did not release the CEL files so we cannot determine if this difference is biological or technical in nature. Using RNA isolated from our adipose tissue samples (10 ng total RNA) the RNA was reverse-transcribed using reverse transcription reagents (Applied Biosystems), according to the manufacturer's protocol to profile microRNAs.

Detection of cDNAs was performed using an ABIPRISM $^{\circledR} 7900$ Sequence Detection system (Applied Biosystems). Primers (Invitrogen) were designed by Primer Express software (Applied Biosystems) or by the Universal Probe Library (Roche Applied Science). A pre-optimized primer and probe assay for $18 \mathrm{~S}$ rRNA was used as an endogenous control (Applied Biosystems). Primers and probes were pre-mixed with TaqMan Universal Master Mix or SYBR ${ }^{\circledR}$ GREEN PCR Master Mix (Applied Biosystems) and applied to 384-well MicroAmp Optical barcode plates (Applied Biosystems). cDNA aliquots of $4 \mu$ l were added in triplicates. The amplification of genomic DNA typically amounted to a maximum of $<1 \%$ of the target gene when extracting RNA by TRIzol [37]. Thermal cycling conditions were: $2 \mathrm{~min}$ at $50^{\circ} \mathrm{C}, 10 \mathrm{~min}$ at $95^{\circ} \mathrm{C}$ and 40 cycles of $15 \mathrm{~s}$ at $95^{\circ} \mathrm{C}$ and $1 \mathrm{~min}$ at $65^{\circ} \mathrm{C}$. Two-fold dilutions series were performed for all target genes and endogenous controls to determine the amplification efficiency.

\section{Gene Ontology analysis using EASE}

A miRNA binds to the 3' UTR of a target mRNA via the "seed" region of the mature miRNA (nt 2-7). For mir-21, we used Gene Ontology ( $\mathrm{GO}$ ) analysis to obtain an overview of the main classes of biological functions of genes predicted to be targets for this miRNA. For miRNA target prediction categorised by Gene Ontology, we used EASE (version 2.0) and a predicted list of conserved miRNA target genes obtained from Targetscan (http://www.targetscan.org/, release 5.0). For the EASE analysis, we enlisted a false discovery rate (FDR) and an EASE score, which we calculated using 500 permutations/iterations. It should be noted that mir-21 is unlikely to be the only obesity regulated miRNA and as such this ontology analysis should be considered hypothesis generating rather than definitive.

\section{Network analysis using PubGene}

The connectivity of putative miRNA targets was analysed using the literature co-citation network PubGene (http://www.pubgene.org). The following explanation is extracted from the PubGene Public Service User Guide. PubGene lists records that co-cite gene identifiers. Cocitation suggests biological relationship between the implicated genes. The Bio Networks tool presents related genes graphically as networks. A count is made of the MEDLINE records where both the query gene and a neighbour appear (or a recognized synonym of either of these). Neighbours of a gene are thereby ranked according to this count. The Bio Associations tool prepares lists of keywords associated with gene or protein terms. Using this tool, we performed a search for process-association with an individual gene ID. The Bio Associations tool returns a list of GO terms for process-associations that co-occur with the query gene in Medline records. The list is ranked depending on scores assigned to each of the co-occurring terms. Scoring was computed by a probabilistic method. Using this method, scores approaching 0 show the strongest association between the query gene and GO term. In principle, scores can be in the range of 0 to 1 . The published formula shows the probability of the term and the gene occurring together the observed number of times under the assumption that their co-occurrence is not meaningful but instead simply a random event (i.e. the probability of the null hypothesis). We utilised this approach as a descriptive tool.

\section{Results}

Identification of adipogenesis-regulated miRNAs in primary white and brown murine adipocytes using miRNA arrays

To examine the role of the post-transcriptional regulators of adipogenesis in a physiologically relevant model, we profiled primary white and brown pre-adipocytes before and after differentiation into mature adipocytes $(\mathrm{n}=3+3$ for each tissue type) using Exiqon miRNA arrays, v.8.1. Data sets were highly consistent both after background subtraction and after quantile normalisation of the background subtracted data. The analysis was performed in two ways. Since there were no large differences between miRNA expression in brown and white adipocytes, we 
used the data from both cell types combined, to increase the sample size and hence statistical power (Additional file 1). 51 unique miRNAs were significantly upregulated and 14 miRNAs were downregulated during the differentiation of both white and brown preadipocytes (FDR $<10 \%$ ). Then we did the analysis of the significant data from brown and white cells separately using a standard Student T-test to look for the differences in miRNA regulation during white and brown adipocyte maturation. While this clearly has limitations, six miRNAs showed a trend for a stronger upregulation during white pre-adipocyte differentiation compared with brown pre-adipocytes, while another 6 miRNAs showed a trend for a stronger upregulation during brown pre-adipocyte development (see Figure 1). Only two miRNAs seemed to be downregulated more strongly during white pre-adipocyte maturation compared to brown, while only one miRNA showed a trend for a stronger downregulation during brown adipocyte differentiation. Such trends are interesting but require confirmation. In general, differences in miRNA regulation between white and brown adipocytes during maturation are not pronounced. The six miRNAs tending to demonstrate a stronger upregulation during the white adipocyte differentiation included miR-24-1* and miR-23b, members of a recently identified miR-23b cluster. Another miRNA that seemed more strongly upregulated during the white adipocyte differentiation was miR-21. This upregulation was not significant in the main analysis (FDR $>10 \%$ ), but we chose to follow up on this miRNA in later experiments because of its reported role in proliferation [38] and inflammation [39]. We also decided to follow up on miR-143, which we found upregulated in both white and brown adipocytes, as mir-143 is induced upon differentiation of human preadipocytes and 3T3-L1 fibroblasts [25-27].

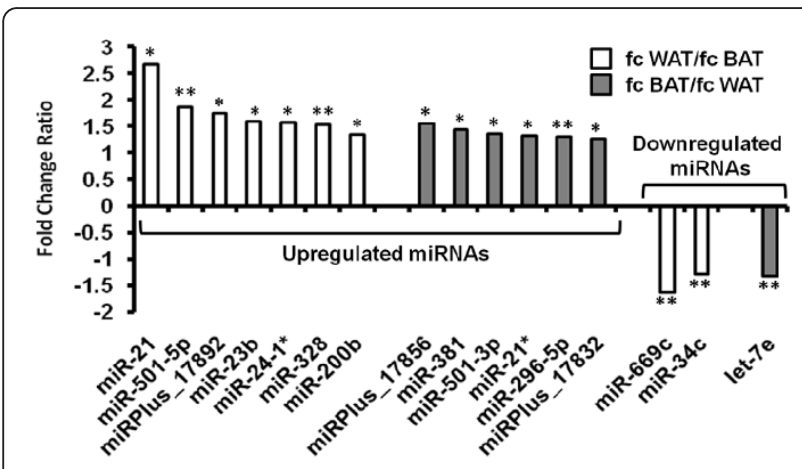

Figure 1 miRNA regulation during adipocyte differentiation Differences between miRNA regulation during differentiation between white and brown adipocytes are shown as a fold difference (ratio) of fold changes. Student T-test was performed to calculate the significance in these differences. ${ }^{*}$ depicts $p<0.1$,

**depicts $p<0.05$.
Six miRNAs showed a trend for a stronger upregulation during the brown adipocyte differentiation - miRPlus_17856, mmu-miR-381, mmu-miR-501-3p, mmumiR-21*, mmu-miR-296-5p and miRPlus_17832. In contrast to the stronger upregulation of miR-21 in white cells, miR-21* (the opposite arm of miR-21) seemed to be more strongly upregulated in brown cells. Of the 14 downregulated miRNAs, miR-669c and miR-34c, tended to be downregulated further during the maturation of white adipocytes compared to browns. One miRNA, mmu-let-7e, seemed more downregulated during the brown adipocyte maturation. Critically, no miRNA showed expression only in browns or whites in this gene chip study, nor did we see an opposite regulation pattern in the two cell types, suggesting that at this level of sensitivity, we captured adipogenesis-regulated miRNAs, rather than miRNAs involved in cell lineage determination.

\section{qPCR profiling of miRNAs in primary white and brown adipocyte cultures}

From the list of the miRNAs that were differentially expressed during adipogenesis of primary white or brown murine adipocytes, we selected a subset of 10 miRNAs for profiling using Taqman-based qPCR. Of these 10 miRNAs, only mir-21, mir34c and mir-143 were differentially expressed between mature adipocytes and preadipocytes $(\mathrm{p}<0.05)$, see Table 1 and Figure 2 . Mir-34c upregulation during brown adipocyte maturation was in contrast to our microarray data and this result must be treated with caution. When comparing the EXIQON chip and the TaqMan assay, mir-34c demonstrated the opposite result suggesting that either the gene-chip probe or the TaqMan assay is unreliable. Mir-136 and mir-718 were not detectable in the adipocyte cultures using the Taqman assays-on-demand, while mir-346, mir-298, mir-330 and mir-501 were expressed at low levels (Ct levels above 33), see Table 1. This suggests that currently there is no gold standard method (when RNA is limiting) to validate miRNA data profiles. It should however be noted that the development of the EXIQON chip has progressed since Version 8 and some of these issues may now be resolved. Nevertheless, the miRNAs mir-34c, mir-143, mir-24, mir-720 and mir-21 showed robust expression in the adipocyte cultures, and these 5 miRNAs were thus profiled in subcutaneous adipose tissue from healthy humans with different BMIs to examine their regulation in adipose tissue expansion.

\section{A weighted context score ranking analysis of the global changes in adipogenesis-induced changes in miRNA expression}

We previously validated a novel ranking system to calculate the collective impact of both upregulated and 
Table 1 MicroRNA Ct levels \pm standard deviation of samples $(n=3)$

\begin{tabular}{ccccc}
\hline miRNA & WAT preadipocytes & WAT adipocytes & BAT preadipocytes & BAT adipocytes \\
\hline miR-21 & $23.2 \pm 0.4$ & $22.3 \pm 0.2$ & $23.9 \pm 0.8$ & $22.2 \pm 0.2$ \\
miR-24 & $25.5 \pm 0.5$ & $25.2 \pm 0.1$ & $25.5 \pm 0.7$ & $24.9 \pm 0.3$ \\
miR-34c & $28.8 \pm 0.1$ & $28.7 \pm 0.4$ & $29.0 \pm 1.2$ & $27.1 \pm 0.2$ \\
miR-136 & $\mathrm{ND}$ & $\mathrm{ND}$ & $\mathrm{ND}$ & $\mathrm{ND}$ \\
miR-143 & $31.6 \pm 0.6$ & $29.4 \pm 0.6$ & $31.8 \pm 1.2$ & $29.3 \pm 0.5$ \\
miR-298 & $33.2 \pm 0.2$ & $33.8 \pm 0.2$ & $33.5 \pm 0.6$ & $33.5 \pm 0.4$ \\
miR-346 & $35.7 \pm 1.1$ & $36.4 \pm 0.8$ & $35.7 \pm 0.6$ & $34.7 \pm 0.4$ \\
miR-501 & $34.5 \pm 0.9$ & $34.4 \pm 0.9$ & $34.6 \pm 0.1$ & $34.4 \pm 0.4$ \\
miR-718 & $\mathrm{ND}$ & $\mathrm{ND}$ & $\mathrm{ND}$ & $\mathrm{ND}$ \\
miR-720 & $22.5 \pm 0.3$ & $22.9 \pm 0.1$ & $23.4 \pm 0.4$ & $22.9 \pm 0.7$ \\
\hline
\end{tabular}

All miRNA assays are pre-developed assays (PDA) from Applied Biosystems. Reference RNAs used for normalisation, snoRNA142, did not show variation between samples. ND: Not detectable. Note that miRNA expression values where $\mathrm{Ct}=>>30$ could indicate that the TaqMan assay is non-functional.

downregulated miRNAs in vivo [19]. We combined the conserved target site criteria from the Targetscan database with cell-specific mRNA expression data [8] and generated a list of genes reliably expressed in adipocytes and that would be potentially regulated by the combined action of all the changing miRNAs. We combined the two non-overlapping lists of up-regulated and down-regulated putative targets of miRNAs as previously described [19] and tested for the targeted biological processes using the ontological analysis (Additional file 2) using the global adipocyte transcriptome as a back-ground. Highly significant enrichment was uniquely found within the first quartile of ranked genes, including transcription regulation $(\mathrm{p}<1.62 \times$ $10^{-6}$ ) and regulation of cellular metabolic processes ( $\mathrm{p}$ $\left.<4.3 \times 10^{-4}\right)$. The fourth quartile of conserved wCCS targets did not show such enrichment and acts as the

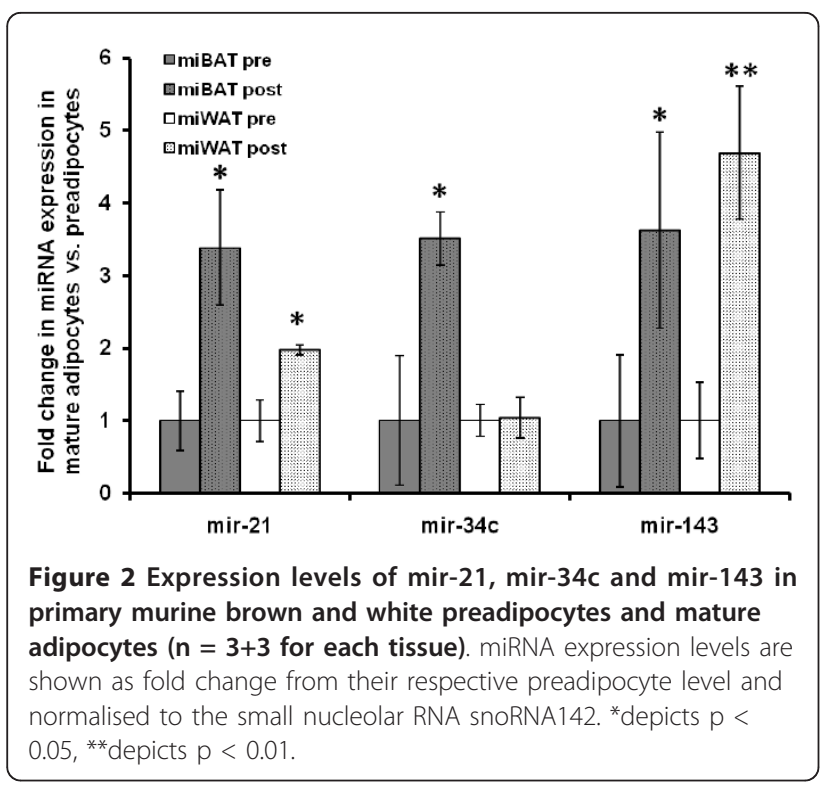

control list for this validated method [19]. Ontological enrichment of target genes shows in a statistically significant manner that distinct biological processes are targeted, notably the ones related to transcription and metabolism even when using only expressed miRNA predicted gene targets (a gene list that has an ontological bias per se).

\section{Network analysis of putative miRNA targets}

The connectivity of first quartile ranked genes belonging to the ontology category SP_PIR_Transcription Regulation genes (FDR $<2 \times 10^{-6}$, in total 37 genes) was analysed using the literature co-citation network PubGene (http://www.pubgene.org). PubGene lists records that co-cite gene identifiers. Co-citation suggests biological relationship between the implicated genes. The network showing the interconnections between analysed genes and their first order literature neighbours is shown in Figure 3. In total there are 132 connections, while the network based on probability of chance co-occurrence contains 67 connections. The most connected gene from this analysed group was Sirt1, which linked directly to 5 other genes from the analysed group (Sox4, Bcl6, Neurod1 and Smad7). Using PubGene process associations, we annotated all 37 genes to biological processes by probabilistic scoring. Five out of 37 analysed genes (Ebf1, Ncoa1, Nr5a2, Sirt1 and Smad7) are involved in fat cell differentiation $\left(\mathrm{p}<1.9 \times 10^{-7}\right)$ (see Additional file 1), while all of the 37 genes play a role in differentiation, development and/or proliferation processes ( $\mathrm{p}<$ 0.001 ). PubGene associated are not fully validated and such association should be taken as hypothesis generating.

\section{Regulation of adipogenesis-regulated miRNAs in obesity} Five miRNAs (mir-21, mir-143, mir-34c, mir-24 and mir-720) were profiled in subcutaneous adipose tissue 


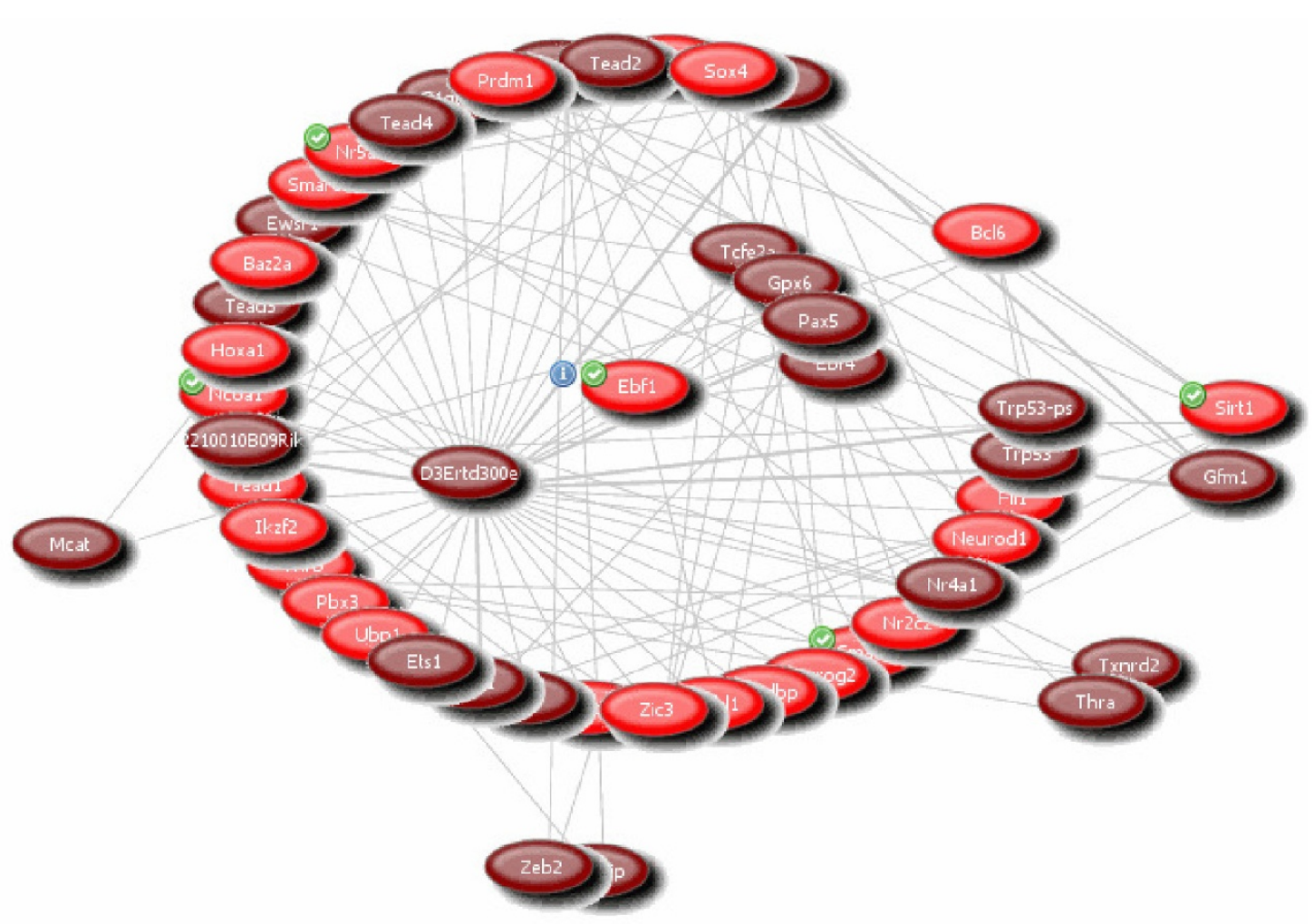

Figure 3 Network showing the interconnections between 1q miRNA targets belonging to GO category "SP_PIR_Transcription Regulation genes" (colored in red) and their first order literature neighbours (colored in brown). The connectivity of putative miRNA targets was analysed using the literature co-citation network PubGene (http://www.pubgene.org), see Materials and Methods.

from healthy humans with varying degrees of obesity. Mir-21 showed higher expression in persons with a BMI $>30$, while mir-143 showed lower expression in persons with a BMI $>30(\mathrm{p}<0.05)$, see Figure 4. There was a strong positive correlation of mir-21 expression in human adipose tissue with BMI $(\mathrm{p}<0.001$, see Figure 5).

\section{Discussion}

We performed global expression profiling of miRNAs before and after the differentiation of primary murine white and brown preadipocytes into mature adipocytes, in order to discover post-transcriptional regulators of adipogenesis. We found 65 miRNAs differentially expressed following differentiation of preadipocytes into adipocytes, but only subtle differences in fold-change of miRNAs between white and brown adipocytes, suggesting that the identified miRNAs most likely play a role in adipogenesis per se rather than in cell lineage determination. Ontological analysis of the target genes of the miRNAs regulated during in vitro adipogenesis indicated that processes related to transcription and metabolism are potentially targeted by the alteration in miRNA expression. One of the identified adipogenesis-regulated
miRNAs, mir-21, showed a positive correlation with obesity in subcutaneous adipose tissue from healthy humans and this merits further investigation to verify the location of mir-21 expression in situ, in adipose tissue.

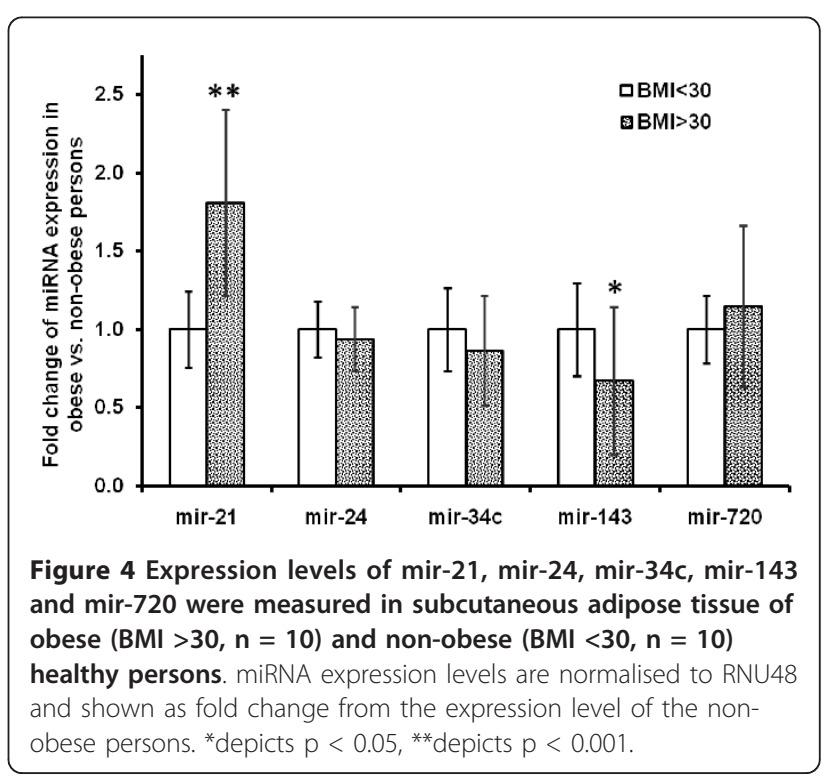




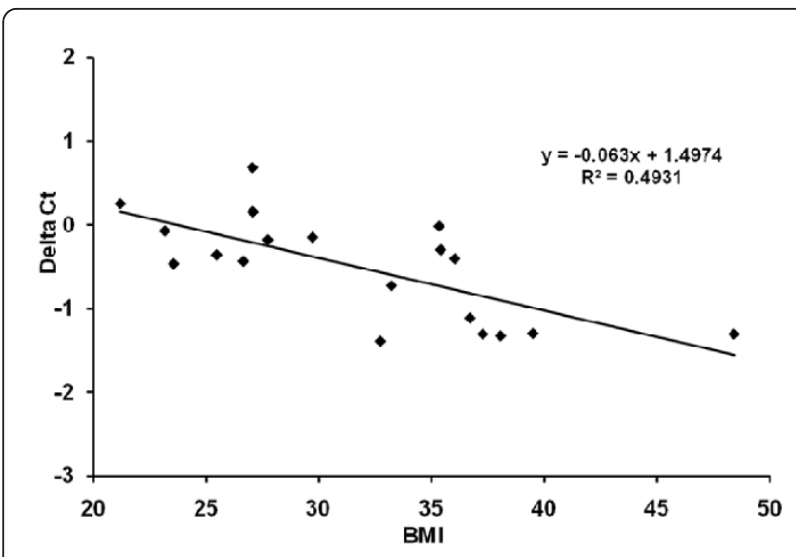

Figure 5 Regression plot of mir-21 expression levels in subcutaneous adipose tissue of healthy persons $(n=20)$ with a range in BMI (21-48). Data are presented as $\Delta \mathrm{Ct}$ levels (difference between $\mathrm{Ct}$ value for miR-21 and $\mathrm{Ct}$ value for control RNU48) versus BMI and thus represents a positive correlation between mir-21 expression levels and BMl, $\mathrm{p}<0.001$.

\section{MiRNAs regulated by adipogenesis}

Fiftyone miRNAs were significantly upregulated and fourteen downregulated during the differentiation of both white and brown preadipocytes. The trend for differences in the degree of miRNA upregulation or downregulation between the white and brown adipocytes was 2 -fold. MiR-143 and miR-181a were expressed in both white and brown adipocytes during both developmental stages and showed a trend for a 2-fold enrichment in white adipocytes, similarly to what was found by Walden et al. [16]. However, from this data set, differences in miRNA regulation between white and brown adipocyte population were very modest. 'Muscle-enriched' miRNAs, miR-1 and miR-133a, previously shown by Walden et al. to be modestly yet expressed in brown versus white adipocytes (but not upregulated during their differentiation), were too low in expression to be detected by this microarray study.

Two of the six miRNAs showed a trend for a stronger upregulation during white adipocyte differentiation miR-24-1* and miR-23b. They belong to a recently identified miR-23b cluster shown to be a molecular switch regulating $\mathrm{TGF}_{\text {万 }}$ response of liver stem cells during their differentiation [40], and also to be regulated by bone morphogenetic protein-2 (BMP-2) in mesenchymal stem cells differentiating into adipocytes [41]. The BMP2 signalling pathway plays a role in white adipocyte lineage determination, as BMP-2 can induce commitment of C3H10T1/2 pluripotent stem cells into white adipocytes [42]. Our results, in combination with these previous observations, suggest that this mechanism might therefore involve miR-24-1 and miR-23b. Another important member of this miR-23b cluster is miR-27b, which has also been shown to play important roles in adipogenesis by impairing differentiation of human adipocytes and targeting PPAR $\gamma$ [43].

However, we found that miR-27b was not robustly expressed in brown or white primary adipocytes. Two miRNA, miR-669c and miR-34c, demonstrated a trend for downregulation during the maturation of white but not brown adipocytes. miR-669c has recently been implicated in ageing [44] and correlates with the decline of liver regeneration. The only miRNA that was significantly downregulated in both brown and white adipocytes (yet more so during the brown adipocyte maturation) was let$7 \mathrm{e}$, which belongs to the let-7 family. Let-7 impairs adipogenic differentiation [29], explaining its downregulation during the adipogenic process. Consistent with in vitro adipogenesis, which consists of two processes - cell proliferation followed by differentiation - most of the miRNAs regulated by adipogenesis in our system are involved in the regulation of these two processes.

\section{Evaluation of the similarity to other models through literature study}

Ortega et al. examined global miRNA expression profiles during human adipogenesis and whether its pattern significantly differed between cells from obese or lean subjects [14]. The differentiation protocol they used differed significantly from ours. They cultured human preadipocytes and passed them through 3 passages before the differentiation was induced at confluency using a cocktail of human Insulin, Dexamethasone, Isobutylmethyl-xanthine (IBMX), and the PPAR $\gamma$ agonist Rosiglitazone, in the presence of fetal bovine serum. This cocktail of drugs is usually necessary to promote successful differentiation in clonal cell lines. The cells we isolated from fat tissues were plated and the differentiation was induced spontaneously in media supplemented with only insulin and newborn bovine serum, and as such it perhaps provides a more native differentiation context. In Ortega's findings most of the miRNAs showed increased or decreased regulation after 7 days of differentiation. They identified the cluster of miRNAs related to miR-30 (miR-30a, b, c, d and e) as being increased during human white adipocyte maturation and these data are in accordance with our observations [14]. Ortega et al., however, show miR-503 as the most down-regulated miRNA during differentiation of human adipocytes [14], while in our murine data set miR-503 was upregulated during both primary brown and white adipocyte differentiation.. The discrepancy with respect to mir-503 regulation may relate to differential regulation between man and mouse, or differences in the differentiation protocols applied.

Our results are also mostly in agreement with those of Esau et al. [25] who identified a similar expression pattern regarding miR-130b, miR-30c, miR-30a*, miR-191, 
miR-30d, miR-196, miR-30b, miR-19b, miR-92, miR-138 and miR-100 during differentiation of cultured human adipocytes. However, our results are not in accordance with Esau regarding miR-20, miR-93, miR-103 and miR107. Esau et al., similarly to Ortega et al., used a cocktail of drugs to induce the differentiation of human preadipocytes. Thus, these discrepancies could again reflect differences between human and mouse adipogenesis or differences in in vitro culture conditions. Xie et al. [27] identified miR-143, miR-148a, miR-30c, miR146b as being upregulated during in vitro differentiation of 3T3L1 cells, which is identical to our findings. However, Xie et al. additionally found miR-107 and miR-103 to be upregulated, similarly to Ortega's findings in human adipocytes, but contrary to our results. As the regulation of mir-107 and mir-103 exists in man and mouse, differences in the differentiation protocols applied could explain the discrepancy. One must also consider the time when such miRNA analysis was run and the possibility of alterations in the annotation of both miRNA sequence and chip design.

One example of miRNAs that can be actively involved in cell proliferation is the miR-17-92 cluster, which comprises seven miRNAs (miR-17-5p, miR-17-3p, miR-18, miR-19a, miR-20, miR-19b, and miR-92-1). This miR-1792 cluster is frequently amplified in B-cell lymphomas and lung cancers, and promotes tumour growth in human and mouse cell models $[45,46]$. Wang et al. used 3T3-L1 preadipocyte cells to screen miRNA expression over seven timepoints after hormonal induction [28]. They found all five members of the miR-17-92 cluster to be markedly upregulated after hormonal stimulation, peaking at the clonal expansion stage. In our dataset, similarly, all members of the cluster were upregulated, suggesting that this cluster might play a role during both proliferation and differentiation of adipocytes.

\section{Predicted protein targets and network analysis}

To better understand the biological consequences of simultaneous changes in multiple miRNAs we utilised a weighted score ranking analysis method [19], which identifies the protein targets most likely to be regulated by collective changes in miRNA expression. We found the ontologies, transcription regulation and regulation of cellular metabolic processes, were strongly represented in the miRNA target list. We made a provisional assessment of the connectivity of the genes belonging to the ontology category 'SP_PIR_Transcription Regulation' using the literature co-citation network PubGene. Sirt1 was linked directly to 5 other genes from the analysed group. Sirt1, an $\mathrm{NAD}^{+}$-dependent deacetylase, belongs to the family of sirtuins, and regulates key aspects of lipid metabolism. Sirt1 plays a role in 3T3-L1 adipogenesis, as Sirt1 protein levels increase during adipogenesis, and overexpression of Sirt1 prevents adipogenesis by inhibiting PPAR $\gamma$ transactivation of adipogenesis-related genes, while knock-down of Sirt1 promotes fat accumulation [47]. Activation of Sirt1 increases the release of FFA in both mature 3T3-L1 adipocytes and in primary white rat adipocytes [47]. Sirt1 also appears to exert an anti-inflammatory effect and improves insulin sensitivity in 3T3-L1 adipocytes by promoting insulin-stimulated glucose uptake [48]. Accordingly, Sirt1 activators improve insulin resistance in ob/ob and diet-induced obese mice, and increase insulin sensitivity in obese, insulin-resistant Zucker rats [49]. Sirt1 genetic variation in humans is related to BMI and risk of obesity [50]. The miRNAs targeting Sirt1 include miR-143, miR-23b miR-34c as well as mir-34a $[51,52]$.

\section{Adipogenesis-regulated miRNAs and human obesity}

Of the miRNAs we described in the murine primary cell cultures, miR-21 and miR-143 were differentially expressed in healthy non-obese persons (BMI <30) versus obese persons $(\mathrm{BMI}>30)$. Furthermore, we found a robust positive correlation of mir-21 with BMI in healthy humans. In the ob/ob mouse, which develops obesity and type 2 diabetes-like symptoms, expression of mir-21 in liver is downregulated [53] suggesting that mir-21 may have different roles depending on the cell type. Both miR-21 and miR-143 show an altered expression level in cancer: miR-21 is upregulated 5-fold in colorectal cancer, when compared with adjacent nontumour tissue, while miR-143 is downregulated 3-fold [54]. In human hepatocellular cancer [55], and in breast tumours [56], miR-21 is also overexpressed, when compared with normal tissue. An anti-miR-21 strategy decreases tumour growth in vivo, possibly by enhancing apoptosis [56]. Cell proliferation is increased by miR-21 overexpression, while it is decreased by an anti-miR-21 strategy, when modest changes in miR-21 expression are achieved (2 to 4-fold changes) [55]. In obese subjects there is an expansion of fat tissue, and while mir- 21 is positively correlated to BMI, it is unknown whether mir-21 can induce adipose tissue expansion, or if it is increased as a consequence of adipose tissue expansion or the inclusion of additional cell types within the tissue. Gabriely et al. found that downregulation of mir-21 in glioma cells reduces their invasive potential, probably by relieving mir-21 targeting of metalloprotease inhibitors, allowing metallo proteases to be active [57]. These data thus suggest that mir-21 could be important for tissue expansion, albeit this will require substantial experimental verification.

\section{Conclusion}

We have identified 65 miRNAs regulated during adipogenesis in primary brown and white adipocytes and 
show that one miRNA, mir-21, correlates positively with human obesity. We also compared our list of adipogenesis-regulated miRNAs with different model systems and find that there are important differences between cell lines or animal models of obesity, and our primary adipocyte profiles. We provide an mRNA and miRNA gene-chip resource for further analysis.

\section{Additional material}

\section{Additional file 1: Significantly upregulated and downregulated miRNAs during the differentiation of both white and brown preadipocytes combined \\ Additional file 2: Gene ontology analysis of the upregulated and downregulated putative targets of miRNAs.}

\section{Acknowledgements}

This study was funded by BBSRC (BB/G013128/1) (BC/JAT). The Chief Scientist Office, Scotland (JT), The Swedish Research Council (BC/JN), the Lundbeck Foundation (PK), and Direktør Emil Hertz and Inger Hertz's Foundation (PK). We thank Dr BK Pedersen for access to human adipose tissue RNA and Dr Amy Buck for assistance with the miRNA profiling.

\section{Author details}

${ }^{1}$ Royal Veterinary College, University of London, UK. ${ }^{2}$ Department of Physiology, The Wenner-Gren Institute, Stockholm University, Sweden. ${ }^{3}$ Tissue Injury \& Repair Group, The Chancellor's Building, University of Edinburgh, EH16 4SB, UK. ${ }^{4}$ Department of Molecular Genetics, Novo Nordisk A/S, DK-2760 Måløv Denmark.

\section{Authors' contributions}

PK carried out the QPCR and EXIQON gene chip analysis. VG performed the PubGene analysis and data mining. VG and PK drafted the manuscript. NP carried out preparation of murine samples for microarray analysis. IJG performed the miRNA target ranking and Gene Ontology analysis. JT, JN and BC conceived the study, and participated in its design, coordination and microarray analysis. All authors edited the article and all authors read and approved the final manuscript.

\section{Competing interests}

The authors declare that they have no competing interests.

Received: 5 October 2010 Accepted: 22 March 2011

Published: 22 March 2011

\section{References}

1. Mokdad AH, Ford ES, Bowman BA, Dietz WH, Vinicor F, Bales VS, Marks JS: Prevalence of obesity, diabetes, and obesity-related health risk factors, 2001. Jama 2003, 289:76-79.

2. Rosen ED, Spiegelman BM: Adipocytes as regulators of energy balance and glucose homeostasis. Nature 2006, 444:847-853.

3. Cannon B, Nedergaard J: Brown adipose tissue: function and physiological significance. Physiol Rev 2004, 84:277-359.

4. Zingaretti MC, Crosta F, Vitali A, Guerrieri M, Frontini A, Cannon B, Nedergaard J, Cinti S: The presence of UCP1 demonstrates that metabolically active adipose tissue in the neck of adult humans truly represents brown adipose tissue. Faseb J 2009, 23:3113-3120.

5. van Marken Lichtenbelt WD, Vanhommerig JW, Smulders NM Drossaerts JM, Kemerink GJ, Bouvy ND, Schrauwen P, Teule GJ: Coldactivated brown adipose tissue in healthy men. N Engl J Med 2009, 360:1500-1508

6. Cypess AM, Lehman S, Williams G, Tal I, Rodman D, Goldfine AB, Kuo FC, Palmer EL, Tseng YH, Doria A, et al: Identification and importance of brown adipose tissue in adult humans. N Engl J Med 2009, 360:1509-1517.
7. Timmons JA, Pedersen BK: The importance of brown adipose tissue. $N$ Engl J Med 2009, 361:415-416, author reply 418-421.

8. Timmons JA, Wennmalm K, Larsson O, Walden TB, Lassmann T, Petrovic N, Hamilton DL, Gimeno RE, Wahlestedt C, Baar K, et al: Myogenic gene expression signature establishes that brown and white adipocytes originate from distinct cell lineages. Proc Natl Acad Sci USA 2007, 104:4401-4406.

9. Tseng YH, Kokkotou E, Schulz TJ, Huang TL, Winnay JN, Taniguchi CM, Tran T, Suzuki R, Espinoza DO, Yamamoto Y, et al: New role of bone morphogenetic protein 7 in brown adipogenesis and energy expenditure. Nature 2008, 454:1000-1004.

10. Seale P, Bjork B, Yang W, Kajimura S, Chin S, Kuang S, Scime A, Devarakonda S, Conroe HM, Erdjument-Bromage H, et al: PRDM16 controls a brown fat/skeletal muscle switch. Nature 2008, 454:961-967.

11. Petrovic N, Walden TB, Shabalina IG, Timmons JA, Cannon B, Nedergaard J: Chronic peroxisome proliferator-activated receptor gamma (PPARgamma) activation of epididymally derived white adipocyte cultures reveals a population of thermogenically competent, UCP1containing adipocytes molecularly distinct from classic brown adipocytes. J Biol Chem 2010, 285:7153-7164.

12. Rosen ED: The transcriptional basis of adipocyte development. Prostaglandins Leukot Essent Fatty Acids 2005, 73:31-34.

13. Seale $P$, Kajimura $S$, Yang W, Chin S, Rohas LM, Uldry M, Tavernier G, Langin D, Spiegelman BM: Transcriptional control of brown fat determination by PRDM16. Cell Metab 2007, 6:38-54.

14. Ortega FJ, Moreno-Navarrete JM, Pardo G, Sabater M, Hummel M, Ferrer A Rodriguez-Hermosa Jl, Ruiz B, Ricart W, Peral B, Fernandez-Real JM: MiRNA expression profile of human subcutaneous adipose and during adipocyte differentiation. PLOS One 2010, 5:e9022.

15. Martinelli R, Nardelli C, Pilone V, Buonomo T, Liguori R, Castano I, Buono P, Masone S, Persico G, Forestieri P, et al: miR-519d Overexpression Is Associated With Human Obesity. Obesity (Silver Spring) 2010.

16. Walden TB, Timmons JA, Keller P, Nedergaard J, Cannon B: Distinct expression of muscle-specific microRNAs (myomirs) in brown adipocytes. J Cell Physiol 2009, 218:444-449.

17. Grimson A, Farh KK, Johnston WK, Garrett-Engele P, Lim LP, Bartel DP: MicroRNA targeting specificity in mammals: determinants beyond seed pairing. Mol Cell 2007, 27:91-105.

18. He A, Zhu L, Gupta N, Chang Y, Fang F: Overexpression of micro ribonucleic acid 29, highly up-regulated in diabetic rats, leads to insulin resistance in 3T3-L1 adipocytes. Mol Endocrinol 2007, 21:2785-2794.

19. Gallagher IJ, Scheele C, Keller P, Nielsen AR, Remenyi J, Fischer CP, Roder K, Babraj J, Wahlestedt C, Hutvagner G, Pedersen BK, Timmons JA: Integration of microRNA changes in vivo identifies novel molecular features of muscle insulin resistance in Type 2 Diabetes. Genome Med 2010, 2:9

20. Bartel DP: MicroRNAs: genomics, biogenesis, mechanism, and function. Cell 2004, 116:281-297.

21. Esau C, Davis S, Murray SF, Yu XX, Pandey SK, Pear M, Watts L, Booten SL, Graham M, McKay R, et al: miR-122 regulation of lipid metabolism revealed by in vivo antisense targeting. Cell Metab 2006, 3:87-98.

22. Poy MN, Eliasson L, Krutzfeldt J, Kuwajima S, Ma X, Macdonald PE, Pfeffer S, Tuschl T, Rajewsky N, Rorsman P, Stoffel M: A pancreatic islet-specific microRNA regulates insulin secretion. Nature 2004, 432:226-230.

23. Plaisance $V$, Abderrahmani $A$, Perret-Menoud $V$, Jacquemin $P$, Lemaigre $F$, Regazzi R: MicroRNA-9 controls the expression of Granuphilin/Slp4 and the secretory response of insulin-producing cells. J Biol Chem 2006, 281:26932-26942.

24. Lovis P, Roggli E, Laybutt DR, Gattesco S, Yang JY, Widmann C, Abderrahmani A, Regazzi R: Alterations in microRNA expression contribute to fatty acid-induced pancreatic beta-cell dysfunction. Diabetes 2008, 57:2728-2736.

25. Esau C, Kang X, Peralta E, Hanson E, Marcusson EG, Ravichandran LV, Sun Y, Koo S, Perera RJ, Jain R, et al: MicroRNA-143 regulates adipocyte differentiation. J Biol Chem 2004, 279:52361-52365.

26. Kajimoto K, Naraba H, Iwai N: MicroRNA and 3T3-L1 pre-adipocyte differentiation. Rna 2006, 12:1626-1632

27. Xie H, Lim B, Lodish HF: MicroRNAs induced during adipogenesis that accelerate fat cell development are downregulated in obesity. Diabetes 2009, 58:1050-1057.

28. Wang Q, Li YC, Wang J, Kong J, Qi Y, Quigg RJ, Li X: miR-17-92 cluster accelerates adipocyte differentiation by negatively regulating tumorsuppressor Rb2/p130. Proc Natl Acad Sci USA 2008, 105:2889-2894. 
29. Sun T, Fu M, Bookout AL, Kliewer SA, Mangelsdorf DJ: MicroRNA let-7 regulates 3T3-L1 adipogenesis. Mol Endocrinol 2009, 23:925-931.

30. Lin Q, Gao Z, Alarcon RM, Ye J, Yun Z: A role of miR-27 in the regulation of adipogenesis. Febs J 2009, 276:2348-2358.

31. Herrera BM, Lockstone HE, Taylor JM, Wills QF, Kaisaki PJ, Barrett A, Camps C, Fernandez C, Ragoussis J, Gauguier D, et al: MicroRNA-125a is over-expressed in insulin target tissues in a spontaneous rat model of Type 2 Diabetes. BMC Med Genomics 2009, 2:54.

32. He A, Zhu L, Gupta N, Chang Y, Fang F: Over-expression of miR-29, highly upregulated in diabetic rats, leads to insulin resistance in 3T3-L1 adipocytes. Mol Endocrinol 2007.

33. Takanabe R, Ono K, Abe Y, Takaya T, Horie T, Wada H, Kita T, Satoh N, Shimatsu A, Hasegawa K: Up-regulated expression of microRNA-143 in association with obesity in adipose tissue of mice fed high-fat diet. Biochem Biophys Res Commun 2008, 376:728-732.

34. Petrovic N, Shabalina IG, Timmons JA, Cannon B, Nedergaard J: Thermogenically competent nonadrenergic recruitment in brown preadipocytes by a PPARgamma agonist. Am J Physiol Endocrinol Metab 2008, 295:E287-296.

35. Scheele C, Nielsen AR, Walden TB, Sewell DA, Fischer CP, Brogan RJ, Petrovic N, Larsson O, Tesch PA, Wennmalm K, et al: Altered regulation of the PINK1 locus: a link between type 2 diabetes and neurodegeneration? Faseb J 2007.

36. Dahlman I, Forsgren M, Sjogren A, Nordstrom EA, Kaaman M, Naslund E, Attersand A, Arner P: Downregulation of electron transport chain genes in visceral adipose tissue in type 2 diabetes independent of obesity and possibly involving tumor necrosis factor-alpha. Diabetes 2006, 55:1792-1799.

37. Timmons JA, Norrbom J, Scheele C, Thonberg H, Wahlestedt C, Tesch P: Expression profiling following local muscle inactivity in humans provides new perspective on diabetes-related genes. Genomics 2006, 87:165-172.

38. Selcuklu SD, Donoghue MT, Spillane C: miR-21 as a key regulator of oncogenic processes. Biochem Soc Trans 2009, 37:918-925.

39. Lu TX, Munitz A, Rothenberg ME: MicroRNA-21 is up-regulated in allergic airway inflammation and regulates IL-12p35 expression. J Immunol 2009, 182:4994-5002

40. Rogler CE, Levoci L, Ader T, Massimi A, Tchaikovskaya T, Norel R, Rogler LE: MicroRNA-23b cluster microRNAs regulate transforming growth factorbeta/bone morphogenetic protein signaling and liver stem cell differentiation by targeting Smads. Hepatology 2009, 50:575-584

41. Sun F, Wang J, Pan Q, Yu Y, Zhang Y, Wan Y, Wang J, Li X, Hong A: Characterization of function and regulation of miR-24-1 and miR-31. Biochem Biophys Res Commun 2009, 380:660-665.

42. Huang H, Song TJ, Li X, Hu L, He Q, Liu M, Lane MD, Tang QQ: BMP signaling pathway is required for commitment of $\mathrm{C} 3 \mathrm{H} 10 \mathrm{~T} 1 / 2$ pluripotent stem cells to the adipocyte lineage. Proc Natl Acad Sci USA 2009, 106:12670-12675.

43. Karbiener M, Fischer C, Nowitsch S, Opriessnig P, Papak C, Ailhaud G, Dani C, Amri EZ, Scheideler M: microRNA miR-27b impairs human adipocyte differentiation and targets PPARgamma. Biochem Biophys Res Commun 2009, 390:247-251.

44. Maes OC, An J, Sarojini H, Wang E: Murine microRNAs implicated in liver functions and aging process. Mech Ageing Dev 2008, 129:534-541.

45. He L, Thomson JM, Hemann MT, Hernando-Monge E, Mu D, Goodson S, Powers S, Cordon-Cardo C, Lowe SW, Hannon GJ, Hammond SM: A microRNA polycistron as a potential human oncogene. Nature 2005 435:828-833.

46. Hayashita $Y$, Osada H, Tatematsu Y, Yamada H, Yanagisawa K, Tomida S, Yatabe $Y$, Kawahara K, Sekido Y, Takahashi T: A polycistronic microRNA cluster, miR-17-92, is overexpressed in human lung cancers and enhances cell proliferation. Cancer Res 2005, 65:9628-9632.

47. Picard F, Kurtev M, Chung N, Topark-Ngarm A, Senawong T, Machado De Oliveira R, Leid M, McBurney MW, Guarente L: Sirt1 promotes fat mobilization in white adipocytes by repressing PPAR-gamma. Nature 2004, 429:771-776.

48. Yoshizaki T, Milne JC, Imamura T, Schenk S, Sonoda N, Babendure JL, Lu JC, Smith JJ, Jirousek MR, Olefsky JM: SIRT1 exerts anti-inflammatory effects and improves insulin sensitivity in adipocytes. Mol Cell Biol 2009, 29:1363-1374.

49. Milne JC, Lambert PD, Schenk S, Carney DP, Smith JJ, Gagne DJ, Jin L, Boss O, Perni RB, Vu CB, et al: Small molecule activators of SIRT1 as therapeutics for the treatment of type 2 diabetes. Nature 2007, 450:712-716.

50. Zillikens MC, van Meurs JB, Rivadeneira F, Amin N, Hofman A, Oostra BA Sijbrands EJ, Witteman JC, Pols HA, van Duijn CM, Uitterlinden AG: SIRT genetic variation is related to BMI and risk of obesity. Diabetes 2009, 58:2828-2834.

51. Yamakuchi $M$, Ferlito $M$, Lowenstein CJ: miR-34a repression of SIRT1 regulates apoptosis. Proc Natl Acad Sci USA 2008, 105:13421-13426.

52. Lee J, Padhye A, Sharma A, Song G, Miao J, Mo YY, Wang L, Kemper JK: A pathway involving farnesoid $X$ receptor and small heterodimer partner positively regulates hepatic sirtuin 1 levels via microRNA-34a inhibition. J Biol Chem 2010, 285:12604-12611.

53. Li S, Chen X, Zhang H, Liang X, Xiang Y, Yu C, Zen K, Li Y, Zhang CY: Differential expression of microRNAs in mouse liver under aberrant energy metabolic status. J Lipid Res 2009, 50:1756-1765.

54. Slaby O, Svoboda M, Fabian P, Smerdova T, Knoflickova D, Bednarikova M Nenutil R, Vyzula R: Altered expression of miR-21, miR-31, miR-143 and miR-145 is related to clinicopathologic features of colorectal cancer. Oncology 2007, 72:397-402

55. Meng F, Henson R, Wehbe-Janek H, Ghoshal K, Jacob ST, Patel T: MicroRNA-21 regulates expression of the PTEN tumor suppressor gene in human hepatocellular cancer. Gastroenterology 2007, 133:647-658.

56. Si ML, Zhu S, Wu H, Lu Z, Wu F, Mo YY: miR-21-mediated tumor growth. Oncogene 2007, 26:2799-2803.

57. Gabriely G, Wurdinger T, Kesari S, Esau CC, Burchard J, Linsley PS, Krichevsky AM: MicroRNA 21 promotes glioma invasion by targeting matrix metalloproteinase regulators. Mol Cell Biol 2008, 28:5369-5380.

\section{Pre-publication history}

The pre-publication history for this paper can be accessed here: http://www.biomedcentral.com/1472-6823/11/7/prepub

\section{doi:10.1186/1472-6823-11-7}

Cite this article as: Keller et al:: Gene-chip studies of adipogenesisregulated microRNAs in mouse primary adipocytes and human obesity. BMC Endocrine Disorders 2011 11:7.

\section{Submit your next manuscript to BioMed Central and take full advantage of:}

- Convenient online submission

- Thorough peer review

- No space constraints or color figure charges

- Immediate publication on acceptance

- Inclusion in PubMed, CAS, Scopus and Google Scholar

- Research which is freely available for redistribution

Submit your manuscript at www.biomedcentral.com/submit
C Biomed Central 